
Inclusive Workplace

\title{
Gerald Chertavian
}

May 24, 2021

2021-04

https://doi.org/10.29338/wc2021-04

The COVID-19 pandemic is the greatest public health emergency in our nation's history, and the resulting economic recession has led to millions of people being unemployed and hundreds of thousands losing their lives. Both these challenges have disproportionally affected those people who were already vulnerable-low-income workers, especially those without equal access to economic opportunity due to their race, gender, education level, or ZIP code. This crisis has demonstrated just how imperative it is that workforce development reshapes the pathways to opportunity in this country.

Investment and expansion in our nation's workforce development system could reverse the impact in communities that have historically been systematically underinvested. Providing a runway for vulnerable Americans to access the skills they need and the opportunities they deserve will prevent them from falling further behind and help them to excel as community members, employees, and entrepreneurs.

There is an enormous opportunity-and responsibility-in front of us. As we rebuild our economy and our country post-COVID, how can we do it in a way that is equitable and inclusive? How can we intentionally create pathways to ensure that those who have been historically marginalized and left behind can access the economic mobility they deserve? What investments and commitments can be made to reskill and upskill the U.S. workforce, promoting greater opportunity and prosperity?

\section{Closing the opportunity divide}

Before the coronavirus struck, millions of young adults in the United States were out of work, out of school, and cut off from any real path to a stable career. At the same time, employers had growing demand for skilled workers that was not being met by traditional education, training, and workforce development programs. This mismatch is both a business problem and a societal problem: an "opportunity divide" that threatens both our nation's economic competitiveness and the very foundation of civil society.

For two decades, Year Up-the organization I founded-has been working to close the opportunity divide by empowering young adults who are disconnected from the economic mainstream to develop 
the skills, experiences, and networks that are necessary to thrive in corporate America. The program provides six months of workforce development training and a six-month internship with an employer partner. Year Up's training is tailored to meet employer demand for skills so that, at the end of the training, these young adults can prove they can be the economic asset that employers need. The results speak for themselves: 90 percent of Year Up students are employed and/or enrolled in postsecondary education within four months of graduation. An independent evaluation in 2018 by the U.S. Department of Labor showed that graduates of Year Up's eight program sites experienced a significant earnings impact of 53 percent wage gain a year postgraduation, compared to the control group, which is larger than similar, rigorously evaluated workforce programs.

In March 2020, as organizations around the country and the world grappled with the pandemic, Year Up made a significant pivot to continue to serve young adults. The decision to move from classroom instruction to virtual learning was difficult-we had to adjust our instruction and coaching model to find new ways to deliver personalized, high-quality support from afar. The strategic decisions we made to transition to virtual have solidified Year Up's efficacy and longevity in a rapidly changing world.

Of course, the challenge of switching to virtual trainings was not unique to Year Up. These shifts are a major challenge for workforce development organizations that have often relied on in-person training to integrate skills training with workplace readiness supports. This Workforce Currents: Leading Workforce Resurgence article will share Year Up's strategies and experiences so that they may be instructive to similar organizations working to create economic opportunity in the current crisis.

\section{Shift to virtual operations}

When it became clear that Year Up's in-person classroom instruction and training model would no longer work for the foreseeable future, we quickly pivoted to a virtual learning model. Within one week, roughly 1,000 staff members were working remotely, and within two weeks more than 4,000 students and interns nationwide were learning and working virtually.

Shifting to virtual operations required close coordination across the entire Year Up organization. Thousands of laptops, hotspots, and headsets were sent to students and interns so that they could participate in virtual learning and internships. A centralized IT help desk provided assistance to staff and program participants who were deploying and learning new technology tools. We launched a COVID-19 website with information for staff and program participants on site closures, scheduling, technology, and role-specific support. Hundreds of staff learned to use video conferencing to engage effectively with their colleagues and students, simultaneously teaching students virtual engagement skills.

With our virtual learning program up and running for almost a year, Year Up is now focused on applying lessons learned during the crisis by enhancing and leveraging the positive aspects of virtual learning. Various program delivery options are being explored to increase effectiveness and scalability-piloting asynchronous models, conducting hybrid online training complemented by in-person sessions, and launching new initiatives to support our graduates entering what will be a difficult job market. 
Looking ahead, Year Up plans to build more partnerships with nonprofits, businesses, and community colleges to continue to scale our impact and ensure that we build the framework to serve our communities equitably and inclusively. We are excited by the opportunities to scale our programs to provide more young adults the knowledge, skills, and confidence they need to build sustainable careers.

\section{Four-step framework for adapting to change}

In the wake of the pandemic, many organizations are wrestling with changes and challenges like the challenges at the Year Up programs. For organizations weighing how best to respond to rapid changes affecting their mission, this four-step framework has helped Year Up focus its efforts during the pandemic:

\section{Respond to market needs}

Make sure your strategy considers changing market conditions and how that might affect your approach to fulfilling your mission. For example, as the coronavirus forced many businesses to shut down and the economy declined sharply, market demand for talent changed dramatically. Many employers that hire Year Up interns and graduates froze all hiring, but others experienced increased demand for workers to support new business requirements, such as roles in customer service support and health care. By working closely with business partners to understand how market forces were affecting their businesses, Year Up was able to identify trends and adapt our programs to support employers' talent needs. As a result, the program's training curriculum will better prepare students for virtual internships, with new emphasis on effective video conferencing, online collaboration, and cloud storage.

\section{Innovate rapidly-adapt and learn}

While it is imperative to move quickly to respond to fast-changing circumstances, it can be exceedingly difficult to determine with certainty what the best course of action will be amid highly fluid circumstances. Sometimes, however, you simply cannot afford to wait to have all the answers before acting. Act decisively, be agile, and adapt as you go.

It is also important to challenge conventional thinking and your traditional models of operating. We may assume that working remotely is inferior to being together in the same location, that meeting online is less effective than in-person, or that virtual learning is a weak imitation of in-person instruction. If we are going to continue serving the people we care about, it's important that we adapt and modify as needed to support our stakeholders/students. While in-person training might have more immediate results, virtual operations allow far greater opportunity for scaling and testing programs. Being agile means challenging long-held beliefs and assumptions. Be sure to keep an open mind as you consider the best course of action.

\section{Collaborate for maximum impact}

Particularly during times of dramatic change and heightened uncertainty, it is important to draw on the insights of a broad range of people to maximize your organization's impact. Start by engaging your staff and key constituencies. As part of that work, make sure to identify those most affected by COVID-19 or any other situation or change you seek to address. 
To gain a better understanding of what our students and interns would need to be able to train and work effectively in a virtual environment, Year Up surveyed 4,000 participants. Their responses gave us important insights into the challenges they were facing, such as limited access to reliable technology, insufficient internet bandwidth, and lack of private space for working from home. That enabled the program to take proactive steps to address issues that would otherwise have undermined the effectiveness of the program changes we implemented. Year Up continues to adjust program delivery based on follow-up surveys and ongoing input from our staff and students.

Year Up also regularly engages with business partners, educational institutions, and nonprofit organizations to share insights and identify opportunities to support one another's objectives. This collaboration has had a major impact on our ability to apply lessons learned during the pandemic to maximize the positive impacts of virtual operations for our students and interns. The program is now able to match interns to companies without being restricted by geography, placing them in remote roles that fit the meet the needs of the student and the employer wherever the jobs are. This new type of support and collaboration is an exciting indicator of what we expect in the coming years.

\section{Respect your values}

In challenging times, we all have an even greater responsibility to live up to our values. As your organization considers how to adapt to enhance your impact during the current crisis, make sure your strategy aligns closely with your mission, culture, and values. Keep the diverse needs of your staff and constituents at the center of your decision-making, bearing in mind that some needs will be clear and others will require closer examination.

As more organizations commit to advance racial and economic justice, making sure that culture, policies, and practices are equitable and inclusive will be increasingly important to any organization's long-term success. This is a constant focus for us at Year Up, both internally and externally. Our executive team is prioritizing changes that ensure we lead the way in becoming an antiracist organization.

We're committed to supporting our partners in this work, too, and we provide comprehensive training for our partners and supervisors to give them best practices for managing opportunity youth, young people (16-24) who are disconnected from school or work. In response to COVID-19, we created a dedicated module to assist supervisors with managing interns remotely, including resources and templates. This year, we also rolled out our first virtual internship supervisor orientation, which is a comprehensive experience to help partner companies effectively supervise our interns, including guidance on inclusivity, specific steps to engage interns to foster a sense of belonging, and resources to help our young adults build their professional networks.

\section{A more just and inclusive America}

Enhancing and expanding workplace development will be essential in enabling the most vulnerable segments of society to participate fully in our nation's economic recovery. As a part of the backbone of strong communities, nonprofits are well positioned to do this work, in close collaboration with 
government, business, and education organizations. It will take all of us committing to this work to create the pathways that young adults deserve.

As a nation and as business leaders, let's commit to creating a more just, more inclusive America where economic, racial, and social justice are a reality-not just an ideal. There's never been more urgency. Our future as a nation depends on it.

This article is part of the Leading Workforce Resurgence series. The views expressed are the author's and do not necessarily represent the views of the Federal Reserve Bank of Atlanta or the Federal Reserve System.

Gerald Chertavian is the chief executive officer and founder of Year Up, a national career program that empowers urban young adults to enter the economic mainstream. 